\title{
Separated Axillary Tuberculous Lymphadenitis: A Case Report
}

\author{
Samer Makki Mohamed Al-Hakkak \\ Department of Surgery, Faculty of Medicine, Jabir Ibn Hayyan Medical University, Najaf City, Iraq
}

Email address:

Sammerhakak1971@yahoo.com

\section{To cite this article:}

Samer Makki Mohamed Al-Hakkak. Separated Axillary Tuberculous Lymphadenitis: A Case Report. Journal of Surgery. Vol. 5, No. 2, 2017, pp. 18-21. doi: 10.11648/j.js.20170502.12

Received: March 3, 2017; Accepted: March 14, 2017; Published: March 27, 2017

\begin{abstract}
Tuberculosis (TB) still accounts for a high burden disease. It has been estimated that one third of the world population is infected with Mycobacterium tuberculosis, the most residing in developing countries. Separated axillary tuberculous lymphadenopathy is rare and described in patients without proof of previous or outstanding tuberculosis anywhere in the body. TB was supposed to be considered in the differential diagnosis of patients who present with axillary lymphadenopathy, especially in the endemic areas of Tuberculosis. Ultrasonography features of the axillary lymph node in our patient were not as helpful in diagnosis as the biopsy of the lymph node. Axillary tuberculous lymphadenitis diagnosis depends on the complete pathological examination. It remains both diagnostic and therapeutic challenge because it mimics other pathologic processes and yields inconsistent physical and laboratory findings. Diagnosis is difficult often requiring biopsy.
\end{abstract}

Keywords: Separated, Axillary Lymphadenopathy, Tuberculosis, Ultrasound, Biopsy

\section{Introduction}

Extra pulmonary TB accounts for about $7-30 \%$ of TB cases and lymphadenitis accounts for $17-43 \%$ of cases. Cervical lymph nodes constitute the most common site of involvement with axillary nodes affected in $3.8-20.3 \%$ of tuberculous lymphadenitis $[1,2]$. Isolated axillary tuberculous lymphadenitis is rare and described in patients without previous or active pulmonary TB and no evidence of the origin of TB detected elsewhere [3, 4]. Tuberculous lymphadenitis presents as a painless, slowly progressive swelling of a single group of nodes and in $85 \%$ of cases involvement is unilateral [5]. It may resemble breast carcinoma or exist both at the same time resulting in diagnostic and therapeutic challenges $[1,6]$. Confirmation of diagnosis is by histology [4]. Tuberculous lymphadenitis is a local manifestation of the systemic disease. [7] It may occur during primary tuberculous infection or as a result of reactivation of dormant foci or direct extension from a contiguous focus. Primary infection occurs on initial exposure to tubercle bacilli. Inhaled droplet nuclei are small enough to pass muco-ciliary defences of bronchi and lodge in terminal alveoli of lungs. The bacilli multiply in the lung which is called Ghon focus. The lymphatics drain the bacilli to the hilar lymph nodes. The Ghon focus and related hilar lymphadenopathy form the primary complex. The infection may spread from primary focus to regional lymph nodes. From the regional nodes, organism may continue to spread via the lymphatic system to other nodes or may pass through the nodes to reach blood stream, from where it can spread to virtually all organ of the body. Hilar, mediastinal and paratracheal lymphnodes are the first site of spread of infection from the lymphatic drainage routes for the lung parenchyma. [8] Cervical tuberculous lymphadenitis may represent a spread from the primary focus of infection in the tonsils. $[9,10]$ Axillary tuberculous lymphadenitis is rare and has major prevalence between 20 and 50 years old.

\section{Case Report}

A case report of a 45-year-old female who presented in the outpatient clinic with a history of swelling in the left axilla of 6 months duration. The swelling gradually increased in size and was painless. There was no history of constitutional symptoms like low grade fever night sweat no cough, anorexia or drenching; no breast changes or left upper limb lesions. Examination revealed a female in good health, left axillary swellings that measured about 
$3 \times 6 \mathrm{~cm}$ in diameter, mildly tender, firm, smooth surfaced, rubbery and mobile; the contra lateral axilla was unremarkable, breasts, thorough examination of both the breast done was unremarkable, upper limbs and other regions were essentially normal. Examination for other lymphadenopathy cervical and inguinal done also no significant.

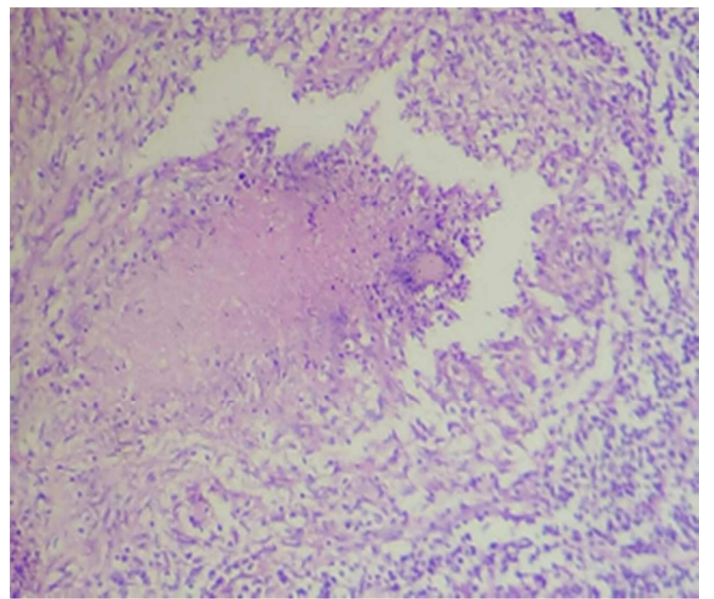

Figure 1. Destruction of lymphoid architecture by multiple granuloma $H \& E \times 40$.

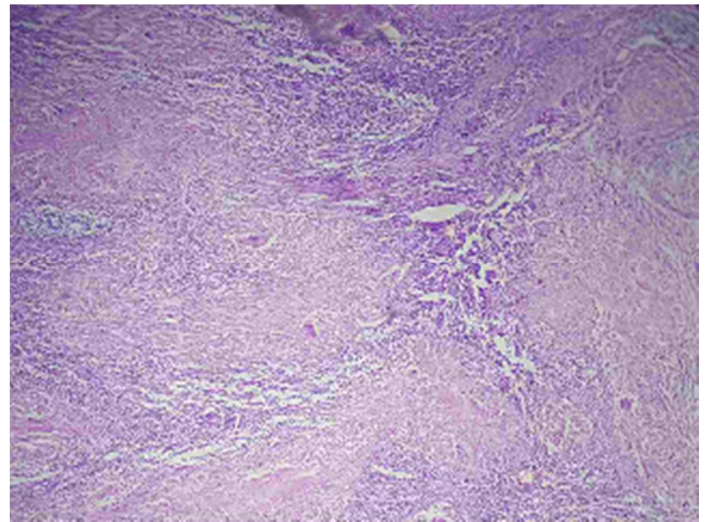

Figure 2. Typical caseating granuloma with presence of multinucleated giant cell $H \& E \times 40$.

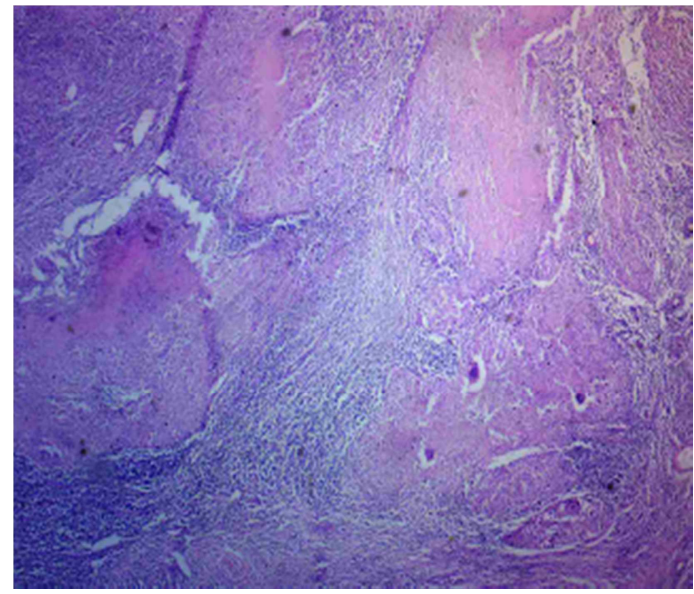

Figure 3. Chronic granulomatous inflammation consistent with tuberculosis $H \& E 100$.
Investigations carried out included complete blood count, hemoglobin $12.5 \mathrm{~g} / \mathrm{dl}$, White blood cell, $6.4 \times 103$ ul: Lymphocytes $40.1 \%$, monocytes $9.9 \%$, granulocytes $50 \%$. Chest X-ray showed clear lung fields; Ultrasound and Duplex is done for both breast and both axilla for assessment of lymphadenopathy which showed left enlargements matted axillary lymphadenopathy with suspicious of secondary metastasis or even lymphoma. Viral screen done for both hepatitis and HIV, So patient proceed for open biopsy, She had left axillary lymph node biopsy; clinical data and gross examination showed single piece of tissue measure $(6 \times 3 \times 2) \mathrm{cm}$ cut section reveled 3 lymph nodes matted together have white firm cut surfaces multiple piece taken for in 3 cassette. Microscopically section reveled destruction of lymphoid architecture by multiple caseation granuloma with presence of multinucleated giant cells. So the diagnosis left axillary lymph nodes with chronic granulomatous inflammation consisted with tuberculosis (figure 1, 2 \& 3). She started anti-tuberculosis chemotherapy with good therapeutic response. two months post chemotherapy the left axilla was decrease size of lymph nodes and about normal size.

\section{Discussion}

Lymphadenitis is the most common clinical presentation of extrapulmonary tuberculosis. Tuberculous lymphadenitis can be local manifestation of the systemic disease. Tuberculous lymphadenitis most frequently involves the cervical lymph nodes (Figure $4 \& 5$ ) followed in frequency by mediastinal, axillary, mesenteric, hepatic portal, perihepatic and inguinal lymph nodes. [11, 12, 13, 14] Extra pulmonary TB incidence had been reported increase in recent years including the subset of lymphadenopathy in keeping with this rare report of an separated axillary lymphadenopathy [2, 5]. Extra pulmonary TB including lymphadenopathy TB being more common, tuberculous lymphadenopathy Axillary tuberculous lymphadenopathy is rare, presents a problem of differential diagnosis with breast carcinoma, and has a major prevalence between 20-50 years old, which was in keeping with our patient aged 45 years [7]. The major diagnostic challenge on clinical evaluation was that she had no history of constitutional symptoms of $\mathrm{TB}$ and no breast lesion on examination and no left limb problems even no underlining skin lesion. The initial thought was that of a lymphadenopathy possibly from lymphoma or an occult breast carcinoma. The breast can be a primary site of TB but more commonly secondary that spreads to the breast through the lymphatic system from the axilla [4]. Our patient may have presented prior to this stage of development. The occurrence of TB with carcinoma is unusual but reported to coexist in axillary lymph nodes without pulmonary or mammary TB [8]. In a situation of diagnostic dilemma, Confirmation of diagnosis is by histology in keeping with our experience (Figure 1, 2 \& 3) [4]. Axillary tuberculosis lymphadenopathy remains a rare condition, and should be considered in patients living in endemic areas of TB. Up to 
$57 \%$ of patients have no systemic symptoms. [9] For 45 years old lady with unilateral axillary tissue biopsy by surgery is advisable. [10, 11, 12].
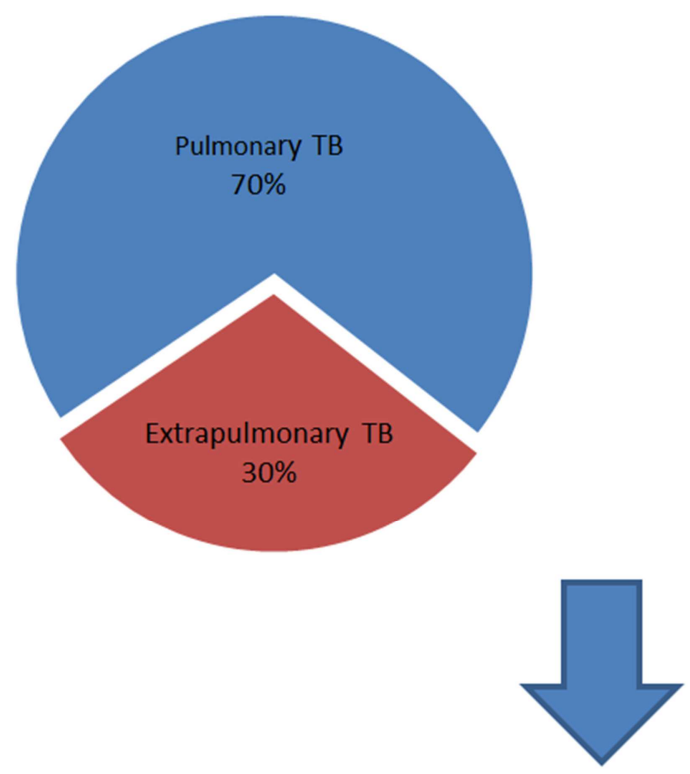

Figure 4. Distrubution of tuberculosis cases.

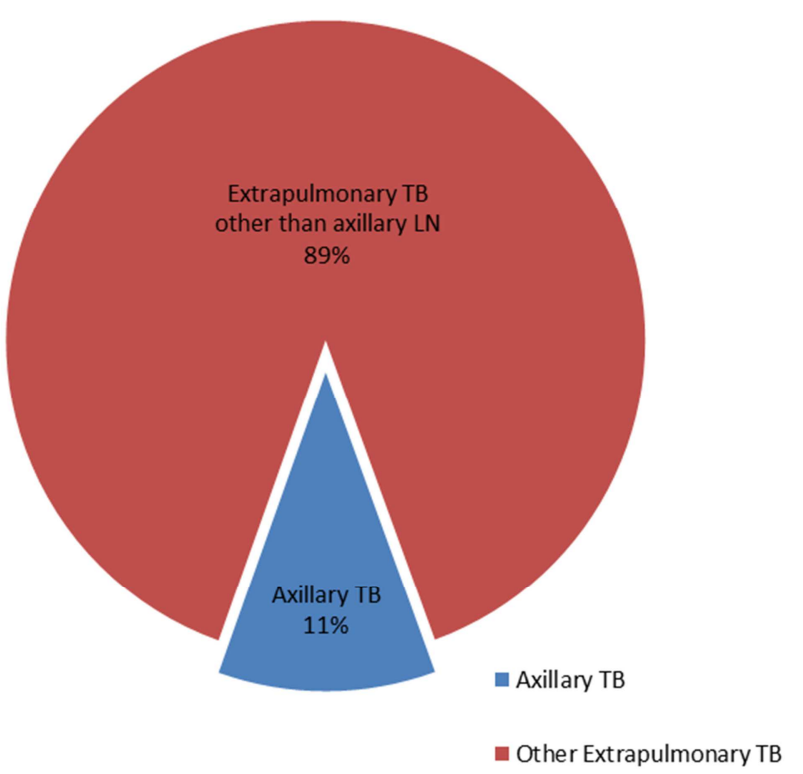

Figure 5. Distrubution of axillary tuberculous lymphadenopathy.

\section{Conclusions}

Tuberculosis is a systemic disease and lymphadenitis is the most common extra-pulmonary manifestation of the disease. Their diagnosis and distinction need a high index of suspicion, and application of suitable diagnostic modalities. The approach to diagnosis should be individualized depending on the location of the disease and the clinical evaluation. Mycobacterial infection should be considered in the differential diagnosis of a axillary swelling, especially in endemic areas. Multiplicity, matting and caseation are three important findings of tuberculous lymphadenitis. Proper judicious use of invasive diagnostic methods and confirmation of the diagnosis. It must be considered about some particular infections like TB during the ultrasonic examinations of swelling lymph nodes in axilla, especially if there is no significant breasts lump.

\section{Acknowledgments}

The author thanks Kaswer Musa Jafaar Al-Tariahi, Ass. Prof. FICMS, from the Department of Pathology and forensic medicine at Kufa Medical College at Najaf City, for providing histology images.

\section{References}

[1] Porto L, Filho IC, Ramalho E, Miranda J, Leal M, et al. Axillary Tuberculous lymphadenitis: a case report and a literature review. Journal of Senologic International Society.

[2] Nwagbara VI, Asuquo ME, Ebughe G, Agbor C, Akpan S, et al. (2013) Tuberculous lymphadenitis of the neck: Case series. International Journam of Medicine 1: 4-8.

[3] Yang CM, Hsu CH, Hsieh CM, Chen MY (2003) 18 F-FDGPET in a clinical unsuspected axillary tuberculosis lymphadenitis mimicking malignancy. Ann Nucl Med Sci 16: 107-110.

[4] Culpinar K, Erpulat Ozis S, Ozdemir S, Korkmaz A (2013) Primary breast tuberculosis: Report of a case. Surgical Science 4: 68-71.

[5] Fontanilla JM, Barnes A, von Reyn CF (2011) Current diagnosis and management of peripheral tuberculous lymphadenitis. Clin Infect Dis 53: 555-562.

[6] Mohapatra PR, Janmeja AK (2009) Tuberculous lymphadenitis. J Assoc Physicians India 57: 585-590.

[7] Chao SS, Loh KS, Tan KK, Chong SM. Tuberculous and non tuberculous cervical lymphadenitis: a clinical review. Otolaryngology Head Neck Surg. 2002; 126: 176-179.

[8] $\mathrm{S}$ hr ine $\mathrm{r} \mathrm{KA}$, Ma thi s en GE, Goe t $\mathrm{z}$ MB. Compa $\mathrm{r}$ i son of mycobacterial lymphadenitis among persons infected with human immunodeficiency virus and seronegative controls. Clin Infect Dis. 1992; 15: 601-605.

[9] Dandapat MC, Mishra BM, Dash SP, Kar PK. Peripheral lymph node tuberculosis: a review of 80 cases. Br J Surg. 1990; 77: 911-912.

[10] Jha BC, Dass A, Nagarkar NM, Gupta R, Singhal S. Cervical tuberculous lymphadenopathy: changing clinical pattern and concepts in management. Postgrad Med J. 2001; 77: 185-187.

[11] Brizi MG, Celi G, Scaldazza AV, Barbaro B. Diagnostic imaging of abdominal tuberculosis: gastrointestinal tract, peritoneum, lymph nodes. Rays. 1998; 23: 115-125.

[12] T hompson MM, Underwood MJ, Sayers RD, Dookeran KA, Bell PR. Peripheral tuberculous lymphadenopathy: a review of 67 cases. Br J Surg. 1992; 79: 763-764.

[13] G eldmacher H, Taube C, Kroeger C, Magnussen H, Kirsten DK. Assessment of lymph node tuberculosis in northern Germany: a clinical review. Chest. 2002; 121: 1177-1182. 
[14] Jerbi M, Hidar S, El Moueddeb S, Jemaa A, Korbi S, et al. (2007) [Tuberculous axillary lymphadenitis: an unusual presentation]. Rev Med Liege 62: 188-189.

[15] Pandey M, Abraham EK, K C, Rajan B (2003) Tuberculosis and metastatic carcinoma coexistence in axillary lymph node: A case report. World J Surg Oncol 1: 3 .

[16] Lee KC, Tami TA, Lalwani AK, Schecter G. Contemporar y management of cervical tuberculosis. Laryngoscope. 1992; 102: 60-64.
[17] Dandapat MC, Mishra BM, Dash SP, Kar PK. Peripheral lymph node tuberculosis: a review of 80 cases. Br J Surg. 1990; 77: 911-912.

[18] Pang SC. Mycobacterial lymphadenitis in Western Australia. Tuber Lung Dis. 1992; 73: 362-367.

[19] Kent DC. Tuberculous lymphadenitis: not a localized disease process. Am J Med Sci. 1967; 254: 866-874. 